\title{
Automated tumor segmentation in MR brain image using fuzzy c-means clustering and seeded region methodology
}

\author{
Mustafa Zuhaer Nayef AL-Dabagh \\ Department of Computer Science, College of Science, Knowledge University, Kurdistan Region, Iraq
}

\begin{tabular}{l} 
Article Info \\
\hline Article history: \\
Received May 12, 2020 \\
Revised Jan 21, 2021 \\
Accepted Feb 2, 2021 \\
\hline
\end{tabular}

\section{Keywords:}

Brain tumor

Magnetic resonance imaging

fuzzy c-means clustering

Morphological operations

Seeds region growing

\begin{abstract}
Automated segmentation of a tumor is still a considerably exciting research topic in the medical imaging processing field, and it plays a considerable role in forming a right diagnosis, to aid effective medical treatment. In this work, a fully automated system for segmentation of the brain tumor in MRI images is introduced. The suggested system consists of three parts: Initially, the image is pre-processed to enhance contrast, eliminate noise, and strip the skull from the image using filtering and morphological operations. Secondly, segmentation of the image happens using two techniques, fuzzy c-means clustering (FCM) and with the application of a seeded region growing algorithm (SGR). Thirdly, this method proposes a post-processing step to smooth segmentation region edges using morphological operations. The testing of the proposed system involved 233 patients, which included 287 MRI images. A comparison of the results ensued, with the manual verification of the traces performed by doctors, which ultimately proved an average Dice Coefficient of $90.13 \%$ and an average Jaccard Coefficient of $82.60 \%$ also, by comparison with traditional segmentation techniques such as FCM method. The segmentation results and quantitative data analysis demonstrates the effectiveness of the suggested system.
\end{abstract}

This is an open access article under the CC BY-SA license.

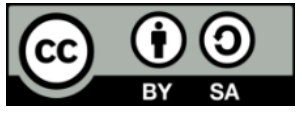

Corresponding Author:

Mustafa Zuhaer Nayef Al-Dabagh

Department of Computer Science

College of Science, Knowledge University

Kurdistan Region, Iraq

Email: mustafa.nayef@knu.edu.iq, mustafa.zuhaer.nayef@gmail.com

\section{INTRODUCTION}

The computer-aided diagnostic system is widely applied to analyze medical images for detection and diagnosis of different kinds of diseases [1]. Brain tumors cause many human deaths, and the early detection and identification of tumors can significantly reduce patient death rates [2]. MRI and calculated tomography (CT) are the most common imaging methods for the diagnosis of cancer. It more accurately determines the tumor location than CT, and it is less harmful than the CT technique because it utilizes a magnetic field as well as radio waves to create itemized images of the brain, while CT uses ionizing radiation [3], [4]. Tumor image segmentation accuracy determines the best segmentation method. Led to its significance, there are a plethora of segmentation methods used today to segment the tumor region of the image [5].

These are a few studies concerning this area, S. Ramathilagam et al. [6] presented a modified robust algorithm based on FCM with a specially weighted bias appreciation for the segmentation of a brain scan using MRI. The algorithm selects the initial centroid using a dist-max initialization method. Applying that clustering validity silhouette method proved that MRFCM-WBE achieved high silhouette values when 
clustering real brain MRI images and lung cancer datasets. It showed to be the most robust method in terms of noise and faster than many other segmentation algorithms. R. Kyle Justice et al. [7] introduced an easy, precise approach to segment MRI brain scan images. That method employed three-dimensional region growing methods guided by changing the number of brain segmentation seed slices. They determined that a growing algorithm employing a 3-D SRG methodology provides a substantial advantage to MRI brain segmentation processes, particularly rapidity and flexibility. Kalaiselvi $\mathrm{T}$ et al. [8] developed a semiautomatic technique for segmentation the brain tumor from T2-weighted images relied on FCM and SRG techniques. That happened by separating the image into four regions and detecting the tumor regions within them. They proved that this technique is more effective and quicker for segmentation the tumor region from T2-weighted images and saves the medical experts diagnosis time. Maksoud et al. [9] developed a hybrid segmentation method, rely on K-means clustering combined with FCM to improve the accuracy, subsequently using thresholding and level set segmentation stages. That method offers more precise results compared to the K-means clustering for image segmentation and improvements in terms of minimizing calculation time. Melegy et al. [10] suggested a new fuzzy logic approach, which is dependent on the FCM algorithm with class center points. That technique applies to MRI images for the automatic segmentation of healthy and patient's brains. The proposed method provides accurate results compared to well-known fuzzy and other favored methodes for MRI segmentation, such as deformable models, and sets a standard for that type of approach. Norhashimah et al. [11] introduced an analysis method of brain tumor segmentation and classification for MR image. To evaluate the proposal method twenty five images were used from BRaTS' 15 . The system had four steps which are preprocessing, segmentation, feature extraction, and classification. The results proved that FCM give good performance which were reached to 0.74 and 0.83 for Jaccard and Dice indices, respectively. Noor et al. [12] suggested a system used image processing for detection and identifying of brain tumor. The system had numbers of step such as pre-processing, edge detection and segmentation. The pre-processing step aim to convert the input image into a greyscale in addition to noise removal. Then, the enhancemnet techniqes were applied to improvment the image. Then it's off the Sobel and Canny algorithm is applied to detect the edge. At last, the segmentation is used to highlight the tumor by using morphological operations.

The major contributions of this work could be outlined:

- $\quad$ In this work, the medical segmentation system relies on fuzzy c-means clustering and seeded region methodologies are introduced to assist medical practitioners to identify the tumor.

- $\quad$ Seeded region methodology is suggested to add before the fuzzy c-means clustering to enhance the segmentation accuracy of fuzzy c-means clustering.

The structure of this paper is: Section 2 demonstrates the suggested approach. Section 3 introduces experimental results. Finally, section 4 gives a conclusion of the work.

\section{PROPOSED APPROACH}

In this proposed approach, two clustering methods are combined to obtain optimally, accurate segmentation results. The proposed system includes of three significant steps: pre-processing, segmentation, and post-processing. In pre-processing, input images are pre-processed to minimize the noise in addition to enhance the contrast between the tumor and its surroundings. Segmentation separates the other tissue from the region of the tumor in the pre-processed image, and finally, in post-processing, the segmentation results are post-processed to remove any other noisy areas. Figure 1 describes the suggested approach.

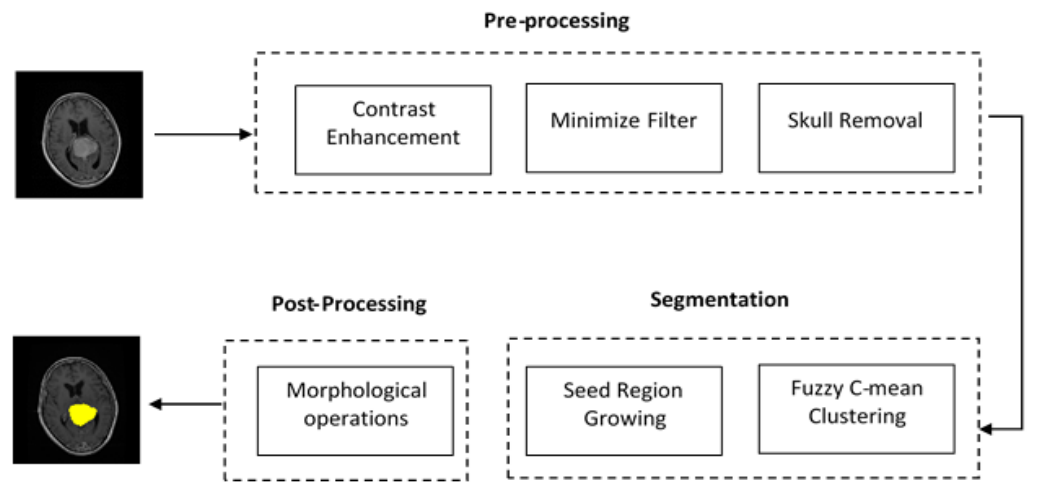

Figure 1. Overview of the suggested approach 


\subsection{Pre-processing}

For the pre-processing stage-Firstly, linear contrast stretching and contrast-enhancing using histogram equalization adjust the image intensity values are applied. Contrast stretching is a simple image enhancemnet method that tries to enhance the contrast in an image by stretching the range of intensity values it includes to obtain full utilization of possible values. The operation of contrast stretching begins by specifying the maximum (a) and minimum (b) values of limits over which image intensity values will be extended. Following, the histogram of the original image is applied to define a minimum value limit (c) and maximum value limit (d) in the original image. Finally, the original valuable $r$ of every pixel is mapped to output variables in (1):

$$
s=(r-c)\left(\frac{b-a}{d-c}\right)+a
$$

Then, histogram equalization is utilized to improve contrast in images through effectively distributing out the most recurrent intensity values. Through this process, the intensities of the image can bestead distributed on the histogram by allowing lower local contrast parts to become a higher contrast without influence on the global contrast. Next, an additional process brightens most of the details in the image except the tumor. A subtraction process then highlights all the objects and their borders in the image, including the tumor. After enhancing the contrast, the minimum filter is applied for three times to replace the pixel intensity with the minimum intensity value. it is a median filter but it replaces a minimum intensity value instead of a median intensity value. Finally, morphological operators on the binary image remove the skull from the image.

\subsection{Segmentation}

This process is aimed to partition the image into a multitude of segments (e.g., sets of pixels or superpixels). Segmentation aims to isolate the lesion(s) from its surrounding pixels and represent that more meaningfully aiding tumor identification by healthcare professionals who can easily differentiate healthy and suspicious regions.

The proposed approach employs FCM clustering then a SRG algorithm. The primary function of the FCM methodology is to find the initial seed, which subsequently is grown by the SGR algorithm, which segments the tumor from that initial seed.

\subsubsection{Fuzzy c-means algorithm (FCM)}

It works unsupervised to correctly cluster processes, analyze features, and classify designs. A diverse number of technological applications use FCM, such as target recognition, geological interpolation, astronomical comparisons, medical diagnostic tools, and image segmentation. Various feature spaces can apply image characterization. The FCM classification algorithm groups comparable image data points from the feature space into clusters. Clustering is achieved by iteratively minimizing a cost function, creating pixel distances, which depend on the domains feature cluster centers in the feature domain [13]. By incessantly optimizing the membership matrix and cluster centers, achieves the extreme point of the objective function, and completes the samples cluster [10]. Considering all currently known fuzzy clustering methods FCM is the most conventional method used to segment the data [14] because it has resilient ambiguity characteristics and can extract much more information than other conventional segmentation methods [15]-[17]. It divides the data set $\mathrm{S}$ to form $\mathrm{L}$ clusters by minimizing the objective function $J_{m}$,

$$
\begin{aligned}
& \min J_{m}=\sum_{k=1}^{L} \sum_{i=1}^{N}\left(\mu_{k i}\right)^{m} d_{k i}^{2}, \\
& \text { s.t. }\left\{\begin{array}{c}
\sum_{k=1}^{L} \mu_{k i}=1 \\
0<\mu_{k i}<1
\end{array}\right.
\end{aligned}
$$

Where $\mu_{k i}$ is the extent that $\mathrm{i}^{\text {th }}$ sample fits within the $\mathrm{k}^{\text {th }}$ cluster, $\mathrm{m}$ is the coefficient that relates to fuzzification $(\mathrm{m} \geq 1, \mathrm{~m} \in \mathbb{R}), \mathrm{L}$ is the number of clusters and exists within the range and conditions stated as $(2 \leq \mathrm{L}<\mathrm{N}, \mathrm{L} \in \mathbb{Z})$. Whereas, the clusters center matrix is defined as $\mathrm{C}=\left[c_{1}, c_{2}, \ldots, c_{L}\right]$ and $d_{k i}=\left\|x_{i}-c_{k}\right\|$ is the Euclidean distance among $x_{i}$ and $c_{k}$. The FCM iterates to produce prototypes and a partition matrix.

$$
\mu_{k i}=\frac{1}{\sum_{f=1}^{L}\left(d_{k i} / d_{f i}\right)^{\frac{2}{m-1}}},
$$




$$
c_{k}=\frac{\sum_{i=1}^{N}\left(\mu_{k i}\right)^{m} x_{i}}{\sum_{i=1}^{N}\left(\mu_{k i}\right)^{m}}
$$

The FCM algorithm is reliant on the definition of some preliminary variables, including the number of clusters (L) and the coefficient of fuzzification $(m)$. Since " $L$ " is an integer and " $\mathrm{m}$ " is a real number, there are countless combinations of "L" and " $m$ " [14].

\subsubsection{Seeded region growing (SRG)}

SGR [18] is a hybrid approach. It commences by recognizing seeds and then expands in those regions by merging pixels into nearest neighbor seed regions [18]. The consideration of two factors must occur during the implementation of SRGS [19]. The first factor focuses on seed point determination to standardly attain seed points positions by Local Maxima Filtering approaches [20], these approaches calculate to determine points with a greater magnitude within a windowed neighborhood [21], [22]. The set of second factors are involved with the similarity index rules; these are used to produce image segments [23]. At this stage, iterative computation continues until all pixels are grouped [24].

A seeded region is grown from a segment into a regional image using a set of q seeds. Given the set of seeds $S_{1}, S_{2}, \ldots, S_{q}$, each phase of SRG takes in one additional pixel to one of the seed sets. Furthermore, these initial seeds are replaced by the centroids of these generated homogeneous regions, $R_{1}, R_{2}, \ldots, R_{q}$, by involving the extra pixels from one stage to the next. The same symbol categorizes the corresponding area's pixels, and different symbols categorize the variant regions pixels. The definition of these categorized pixels is allocated pixels; conversely, the remaining are unallocated pixels. Considering that $\mathrm{H}$ is the group of all unallocated pixels that are adjacent to one or more of the categorized regions.

$$
H=\left\{(x, y) \notin \bigcup_{i=1}^{q} R_{i} \mid N(x, y) \cap \bigcup_{i=1}^{q} R_{i} \neq \emptyset\right\}
$$

Where $N(x, y)$ is the $2^{\text {nd }}$ order neighborhood of the pixel $(x, y)$ as shown in the Figure 2:

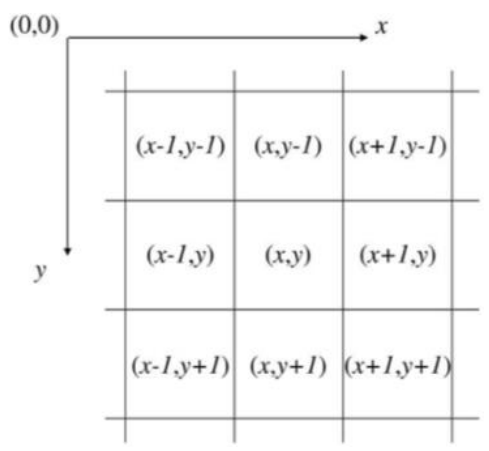

Figure 2. Conventional indexing method

For the uncategorized pixel $(x, y) \in H, N(x, y)$ meets just one of the categorized image regions $R_{i}$ and define $\varphi(x, y) \in\{1,2, \ldots, q\}$ to be that index such that the definition: $\mathrm{N}(\mathrm{x}, \mathrm{y}) \cap \mathrm{R}_{\varphi(\mathrm{x}, \mathrm{y})} \neq \emptyset$. $\delta\left(\mathrm{x}, \mathrm{y}, R_{i}\right)$ is the change among the test pixel at $(x, y)$ and its adjacent categorized region $R_{i} . \delta\left(x, y, R_{i}\right)$, by calculation as:

$$
\delta\left(x, y, R_{i}\right)=\left|g(x, y)-g\left(X_{i}^{c}, Y_{i}^{c}\right)\right|
$$

Where $g(x, y)$ designates the RGB components of the pixel being tested at its coordinate point $(x, y)$; the $g\left(X_{i}^{c}, Y_{i}^{c}\right)$ designation of accounts for average RGB within the homogeneous region $R_{i}$, with $\left(X_{i}^{c}, Y_{i}^{c}\right)$ being the centroid of $R_{i}$. If $N(x, y)$ converges with two or more categorized regions, then $\varphi(x, y)$ is assigned a value of i such that $N(x, y)$ meets $R_{i}$ and minimises $\delta\left(x, y, R_{i}\right)$.

$$
\varphi(x, y)=\min _{(x, y) \in H}\left\{\delta\left(x, y, R_{j}\right) \mid j \epsilon\{1, \ldots, q\}\right.
$$


This SRG procedure repeats to allocate all image pixels to the relevant regions. In (1) and (3) both guarantee the optimally homogeneous separation of the final image partition into a set of regions considering the predefined constraints. The SRG algorithm has no tuning parameters making its implementation robust; it rapidly calculates to perform its task. Those implementation factors make this algorithm desirable for the segmentation of the semantic image. However, SRG algorithm has limitations that pertain to automatic seed selection and pixel sorting [25].

\subsection{Post-processing}

Morphological operations are post-processing steps to smooth the edges of the segmentation region. The main reason for this step is to improve the system performance and provide the image of the brain with a visually clear representation of the tumor area. The application of morphological operations happens using two types of processes: 1) Opening; and 2) Closing. These two morphological operations are implemented based on erosion and dilation operations.

Opening morphological is the first operation applied, smooths the contour of an object, and removes thin portions. Described in the (9) is the mathematical expression of an opening operation:

$$
A \circ B=(A \ominus B) \oplus B
$$

Closing morphological operation is also used to smooth sections of contours but is the opposite of an opening operation; it generally fuses small breaks. Described in the (10) is the mathematical expression of a closing operation:

$$
A \cdot B=(A \oplus B) \ominus B
$$

\section{EXPERIMENTS AND DISCUSSION}

The proposed approach implemented uses MATLAB 2018a. All the experimentation implemented using a personal computer with Core i5-2540M CPU $2.6 \mathrm{GHz}$ and $8 \mathrm{~GB}$ of memory using Windows 7 operating system. The test to prove the effectiveness of the suggested approach involved a dataset [26], [27] of brain T1-weighted CE-MRI images, which contain from 287 selected slices from 233 patients. These are publicly available representative slices that have large lesion sizes. Radiologists have manually delineated each slice to define the tumor boundary. Figure 3 gives a comparison between the results of the suggested approach to the traditional FCM method. Figure 3(a) is shown the original image without any operation while Figure 3(b) is displayed the image after applied pre-processing stage and remove the skull. Figure 3(c) and Figure 3(d) is shown the output image of both the original FCM algorithm and proposal algorithm.

Quantitative evaluation of the proposed methods applies two important statistical measurement parameters: Dice [28] similarity coefficient and Jaccard [29] similarity. The performance measures are defined as (11) and (12):

$$
\begin{aligned}
& \text { Dice }=\frac{2 *|A \cap B|}{|A|+|B|} \\
& \text { Jaccard }=\frac{|A \cap B|}{|A \cup B|}
\end{aligned}
$$

Where A and B are the two binary images.

The segmentation achieved by this approach gives better results than traditional FCM methods, considering efficiency and consistency. Considering the above statistical measure parameters, Table 1 introduces a comparative analysis of the proposed approach, which uses traditional FCM methods. Notably, this table shows that this approach produces few misclassifications, since the number of true negatives is very high, and the quantity of false positives is minimal compared to the FCM algorithm.

Table 1. Comparison between suggested method and different segmentation methods

\begin{tabular}{ccc}
\hline Parameter & Dice Coefficient & Jaccard Coefficient \\
\hline FCM & 0.6864 & 0.5515 \\
Proposal Method & 0.9013 & 0.8260 \\
\hline
\end{tabular}



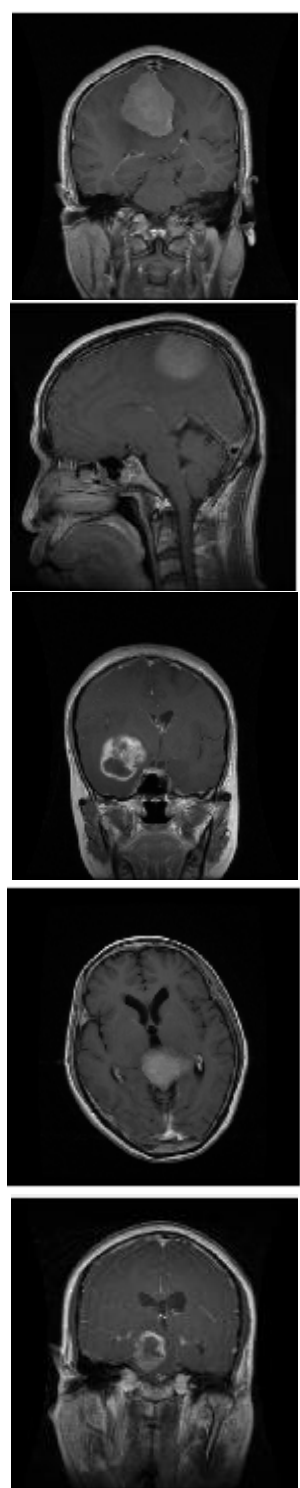

(a)
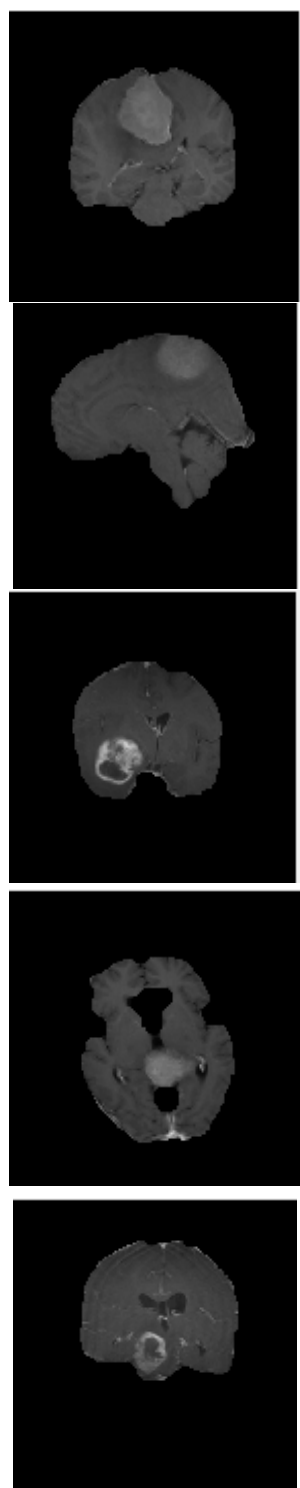

(b)
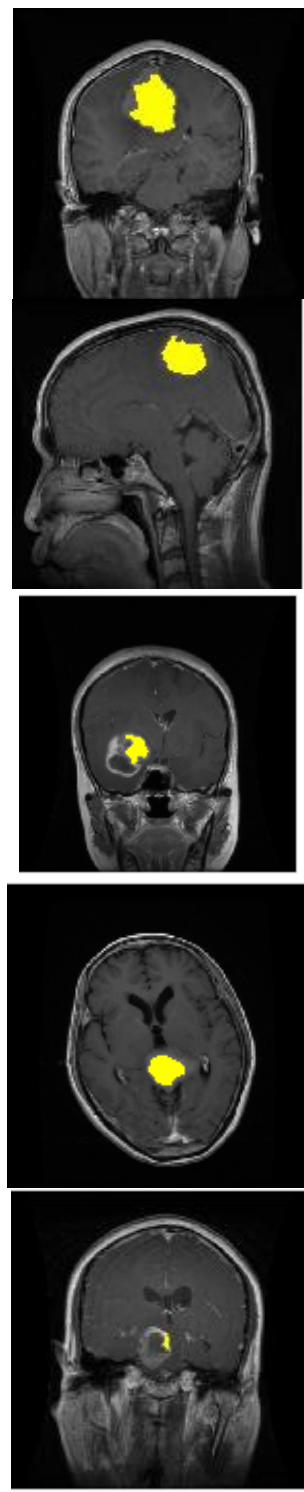

(c)
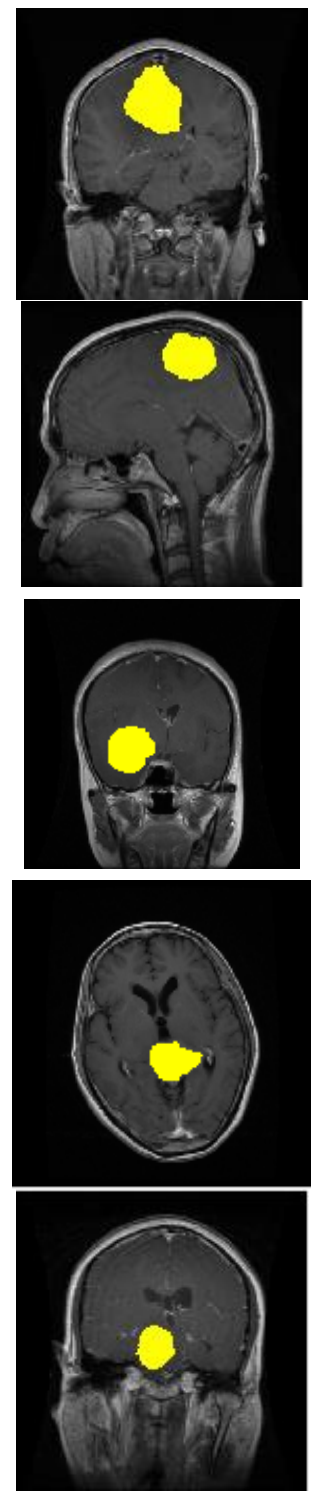

(d)

Figure 3. Results of some successful tumor segmentation, (a) original image [26], [27], (b) Original image after skull removal[26], [27], (c) Segmentation result of FCM[26], [27], (d) Segmentation result of suggested method [26], [27].

\section{CONCLUSION}

In the last decade, MRI has become an imperative tool to aid examination and assist brain tumor diagnosis. MRI is advantageous since it produces high-resolution images of soft tissues without causing radioactive damage to the human body. An accurate brain tumor segmentation method is vital for a patient to get the right treatment from a surgeon. Many conducted studies look at brain tumor segmentation; improving the accuracy of the segmentation method stills a challenging task. This work proposes an automatic approach to detect brain tumors in MRI images using FCM and SGR. Four different methods are applied for speckle noise reduction and construct enhancement such as linear contrast stretching, contrast-enhancing using histogram equalization, subtraction process, and minimum filter. Brain tumor segmentation employs FCM and SGR, accompanied by Morphological operations as post-processing action to smooth the edge of the segmentation region. The segmentation accuracy computes using Dice similarity coefficient and Jaccard similarity through calculates the average for all image results using measured parameters. By experimentation, the proposed approach effectively segments the brain tumor in MRI images and provides a more accurate segmentation result compared to traditional FCM methods. Future accuracy improvements may involve increasing the number of images in the dataset using a pixel-based segmentation approach. 


\section{REFERENCES}

[1] A. R. Ali, M. Couceiro, A. Anter, and A. E. Hassanien, "Particle swarm optimization based fast fuzzy c-means clustering for liver ct segmentation," Intell. Syst. Ref. Libr., 2016, doi: 10.1007/978-3-319-21212-8_10.

[2] A. Chaddad, "Automated feature extraction in brain tumour by magnetic resonance imaging using gaussian mixture models," International Journal of Biomedical Imaging, vol. 2015, pp.11, 2015.

[3] C. P. Samjith Raj and R. Shreeja, "Automatic brain tumor tissue detection in T-1 weighted MRI," in Proceedings of 2017 International Conference on Innovations in Information, Embedded and Communication Systems, ICIIECS 2017, 2018, doi: 10.1109/ICIIECS.2017.8276094.

[4] A. R. Mathew and P. B. Anto, "Tumor detection and classification of MRI brain image using wavelet transform and SVM," in Proceedings of IEEE International Conference on Signal Processing and Communication, ICSPC 2017, 2018, doi: 10.1109/CSPC.2017.8305810.

[5] R. C. Gonzalez and R. E. Woods, Digital Image Processing (3rd Edition). 2007.

[6] S. Ramathilagam, R. Pandiyarajan, A. Sathya, R. Devi, and S. R. Kannan, "Modified fuzzy c-means algorithm for segmentation of T1-T2-weighted brain MRI," Journal of Computational and Applied Mathematics, vol. 235, no. 6, pp. 1578-1586, 2011, doi: 10.1016/j.cam.2010.08.033.

[7] R. K. Justice and E. M. Stokely, "3-D segmentation of MR brain images using seeded region growing," in Annual International Conference of the IEEE Engineering in Medicine and Biology - Proceedings, 1996, doi: 10.1109/iembs.1996.652719.

[8] T. Kalaiselvi, P. Nagaraja, "Brain Tumor Segmentation of MRI Brain Images through FCM clustering and Seeded Region Growing Technique", International Journal of Applied Engineering Research, Research India Publications, Vol.10, No.76, pp.427-432, 2015.

[9] E. Abdel-Maksoud, M. Elmogy, and R. Al-Awadi, "Brain tumor segmentation based on a hybrid clustering technique," Egypt. Informatics J., 2015, doi: 10.1016/j.eij.2015.01.003.

[10] M. T. El-Melegy and H. M. Mokhtar, "Tumor segmentation in brain MRI using a fuzzy approach with class center priors,” Eurasip J. Image Video Process., 2014, doi: 10.1186/1687-5281-2014-21.

[11] N. M. Saad, M. F. Yaakub, A. R. Abdullah, N. S. M. Noor, N. A. Zainal, and W. H. M. Saad, "Automated brain tumor segmentation and classification for MRI analysis system," Indones. J. Electr. Eng. Comput. Sci., 2019, doi: 10.11591/ijeecs.v15.i3.pp1337-1344.

[12] N. E. A. Khalid, M. F. Ismail, M. A. A. B. Manaf, A. F. A. Fadzil, and S. Ibrahim, "MRI brain tumor segmentation: A forthright image processing approach," Bull. Electr. Eng. Informatics, 2020, doi: 10.11591/eei.v9i3.2063.

[13] K. S. Chuang, H. L. Tzeng, S. Chen, J. Wu, and T. J. Chen, "Fuzzy c-means clustering with spatial information for image segmentation," Comput. Med. Imaging Graph., 2006, doi: 10.1016/j.compmedimag.2005.10.001.

[14] H. Yin, Y. Zhang, and Z. Peng, "Optimal sensor placement based on fuzzy c-means clustering algorithm," in Proceedings - 2018 International Conference on Sensor Networks and Signal Processing, SNSP 2018, 2019, doi: 10.1109/SNSP.2018.00027.

[15] J. C. Bezdek, Pattern Recognition with Fuzzy Objective Function Algorithms. 1981.

[16] D. L. Pham and J. L. Prince, "An adaptive fuzzy c-means algorithm for image segmentation in the presence of intensity inhomogeneities," Pattern Recognit. Lett., 1999, doi: 10.1016/S0167-8655(98)00121-4.

[17] J. Hu, M. Wu, X. Chen, S. Du, W. Cao, and J. She, "Hybrid modeling and online optimization strategy for improving carbon efficiency in iron ore sintering process," Inf. Sci. (Ny), 2019, doi: 10.1016/j.ins.2019.01.027.

[18] R. Adams and L. Bischof, "Seeded Region Growing," IEEE Trans. Pattern Anal. Mach. Intell., 1994, doi: 10.1109/34.295913.

[19] F. Y. Shih and S. Cheng, "Automatic seeded region growing for color image segmentation," Image Vis. Comput., 2005, doi: 10.1016/j.imavis.2005.05.015.

[20] M. Erikson, "Segmentation and Classification of individual tree crowns," Swedish University of Agricultural Sciences, Uppsala, Sweden. 2004.

[21] J. Pitkänen and M. Maltamo, "Adaptive Methods for Individual Tree Detection on Airborne Laser Based Canopy Height Model," Int. Arch., 2004.

[22] K. Dralle and M. Rudemo, "Stem number estimation by kernel smoothing of aerial photos," Can. J. For. Res., 1996, doi: 10.1139/x26-137.

[23] M. A. Wulder, J. C. White, K. O. Niemann, and T. Nelson, "Comparison of airborne and satellite high spatial resolution data for the identification of individual trees with local maxima filtering," Int. J. Remote Sens., 2004, doi: 10.1080/01431160310001659252.

[24] T. Kim, C. Ahn, and O. Lee, "Image segmentation by graph cut for radiation images of small animal blood vessels," Microsc. Res. Tech., 2018, doi: 10.1002/jemt.23154.

[25] J. Fan, G. Zeng, M. Body, and M. S. Hacid, "Seeded region growing: An extensive and comparative study," Pattern Recognit. Lett., 2005, doi: 10.1016/j.patrec.2004.10.010.

[26] J. Cheng et al., "Enhanced performance of brain tumor classification via tumor region augmentation and partition," PLoS One, 2015, doi: 10.1371/journal.pone.0140381.

[27] J. Cheng et al., "Retrieval of Brain Tumors by Adaptive Spatial Pooling and Fisher Vector Representation," PLoS One, 2016, doi: 10.1371/journal.pone.0157112.

[28] L. R. Dice, "Measures of the Amount of Ecologic Association between Species," Ecology, 1945, doi: $10.2307 / 1932409$.

[29] P. Jaccard, "The Distribution of The Flora In The Alpine Zone.," New Phytol., 1912, doi: 10.1111/j.14698137.1912.tb05611.x 